\title{
A Comparative Study of Stability Margins for Walking Machines
}

\author{
Elena Garcia Joaquin Estremera Pablo Gonzalez de Santos \\ Instituto de Automatica Industrial. La Poveda, 28500 Madrid, Spain \\ Phone. +34-918711900 FAX. +34-918717050 \\ email: egarcia@iai.csic.es
}

\begin{abstract}
Several static and dynamic stability criteria have been defined in the course of walking robot history. Nevertheless, different applications may require different stability criteria and, to the authors' best knowledge, there is no qualitative classification of such stability measurements. Using the wrong stability criterion to control a robot gait may prevent the task from succeeding. Furthermore, if the optimum criterion is found, the robot gait can also be optimized. In this paper, the stability criteria that have been applied to walking robots with at least four legs are examined in terms of their stability margins in different static and dynamic situations. As a result, a qualitative classification of stability criteria for walking machines is proposed so that the proper criterion can be chosen for every desired application.
\end{abstract}

\section{Keywords}

Walking robot, stability margin, static stability, dynamic stability. 


\section{INTRODUCTION}

Research on walking-robot stability began in 1968, when McGhee and Frank first defined the static stability of an ideal walking robot ${ }^{1}$. Following their definition, an ideal robot is statically stable if the horizontal projection of its center of gravity lies inside the support pattern. The ideal robot is supposed to have massless legs, and system dynamics are assumed to be absent.

The idea of static stability was inspired by insects, whose massless legs must support their body during walking and at the same time provide propulsion. For this reason, their sequence of steps must ensure their static stability. The first generation of walking machines emulated this mechanism of locomotion ${ }^{2}$. These robots were huge mechanisms featuring heavy limbs too difficult to control ${ }^{3}$. The adoption of statically stable gaits could simplify their control. However, during the motion of the heavy limbs and body some inertial effects and other dynamic components (friction, elasticity, etc.) were found to arise, restricting the robot's movements to low, constant velocities. Thus, the adoption of static stability limited these robots' speed of motion.

In the last two decades the walking-robots community has displayed increasing interest in the field of biped robots. Research on dynamic stability has focused on this particular design 4, 5, 6, 7, 8, 9 . Although some dynamically stable quadrupeds exist, they are very mechanically simplified machines having only a few degrees of freedom, which adopt the stability criteria designed for bipeds, extended to a couple of legs $10,11,12$. The motion of these quadrupeds is limited to even terrain, because the stability criterion used (Zero Moment Point) is only valid for that kind of surface $13,14,15$.

Little effort has been made to cope with the dynamic effects that limit statically stable machines' performance $16,17,18,19,20$. However, one of the main goals of research on legged locomotion is the application of walking robots in industrial processes and services, and such robots are not meant to trot or gallop but to walk.

The few dynamic stability criteria defined for quadrupedal walking seem to give different forms and names to a single idea: the sign of the moment around the edges 
of the support polygon caused by dynamic effects acting on the vehicle's center of mass. The suitability of each criterion for each particular application (i.e. manipulation forces and moment present, uneven terrain, etc.) is not clear at all. Nevertheless, the use of a stability criterion not suitable for the current application may prevent the task from succeeding. Therefore, a qualitative classification of the existing static and dynamic stability criteria for robots of four or more legs is absolutely required.

Furthermore, if the optimum criterion were found for each application, robot speed could be increased. Moreover, if some velocity-optimization technique were used for the leg's transfer phase 21,22 , overall machine performance could be optimized.

In this paper, the existing stability criteria are briefly reviewed in Section 2. Then, a comparative study of their stability margins is carried out through simulation using a quadruped robot as testbed in different static and dynamic situations. The simulation features are described in Section 3 and a comparative study on stability margins according to their suitability for measuring stability for a number of representative situations is shown in Section 4. Also, the final qualitative classification of the stability criteria is proposed. Finally, Section 5 presents some conclusions.

\section{A SURVEY ON STABILITY CRITERIA}

\subsection{Static Stability Margins}

The first static stability criterion for an ideal machine walking at constant speed along a constant direction and over flat, even terrain was proposed by McGhee and Frank ${ }^{1}$. The Center of Gravity Projection Method claims that the vehicle is statically stable if the horizontal projection of its center of mass (c.o.m.) lies inside the support polygon (defined as the convex polygon formed by connecting footprints). Later this criterion was extended to uneven terrain ${ }^{23}$ by redefining the support polygon as the horizontal projection of the real support pattern. The Static Stability Margin, SSM, was defined for a given support polygon as the smallest of the distances from the c.o.m. projection 
to the edges of the support polygon. The SSM is the optimum stability margin for an ideal machine on horizontal, even terrain. However, the equation for calculating the SSM is complex. Thus, Zhang and Song proposed the Longitudinal Stability Margin, LSM, defined as the smallest of the distances from the c.o.m. projection to the front and rear edges of the support polygon along the machine's longitudinal axis 24 . The LSM is a good approximation to the SSM, and it is simpler to calculate.

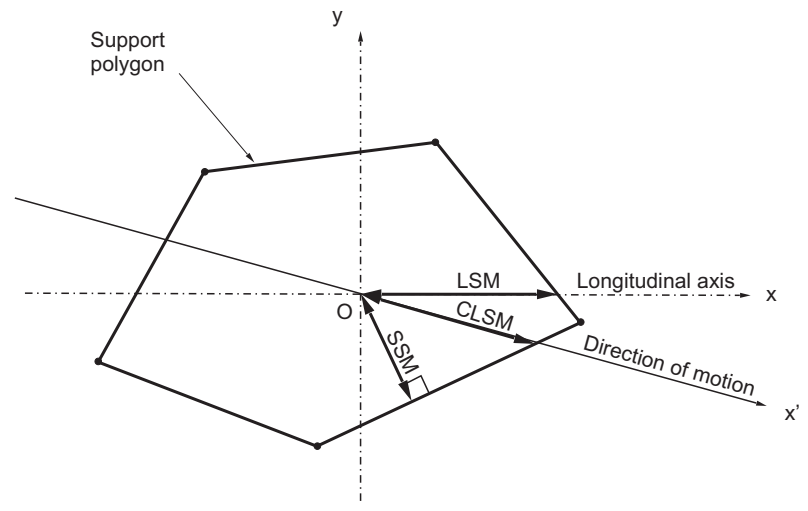

Figure 1: Support polygon and different static stability margins.

Considering the walking robot as a non-ideal vehicle where inertial effects arise during acceleration, the use of the Crab Longitudinal Stability Margin, CLSM, ${ }^{25}$ will be more convenient. The CLSM is the smallest of the distances from the c.o.m. projection to the front and rear edges of the support polygon along the machine's motion axis. Figure 1 shows the SSM, LSM and CLSM for a given support polygon.

Mahalingham et al. ${ }^{26}$ define the Conservative Support Polygon, CSP, as a subset of the support polygon, in order to limit the motion of the c.o.m. projection to guarantee system stability in the case of failure of any of the supporting legs. However, the use of the CSP is restricted to machines with six or more legs using a crawl gait.

The above stability criteria are all based on geometric concepts; the SSM, LSM and CLSM are independent of c.o.m. height and consider neither kinematic nor dynamic parameters. Intuitively speaking, the stability of a non-ideal walking machine should depend on those parameters. 
A better stability measurement was proposed by Messuri 27 . He defined the Energy Stability Margin, ESM, as the minimum potential energy required to tumble the robot around the edges of the support polygon, that is:

$$
S_{E S M}=\min _{i}^{n_{S}}\left(m g h_{i}\right)
$$

where $i$ denotes the segment of the support polygon considered the rotation axis, $n_{s}$ is the number of supporting legs, and $h_{i}$ is the variation of c.o.m. height during the tumble, which comes from:

$$
h_{i}=\left|\mathbf{R}_{\mathbf{i}}\right|(1-\cos \theta) \cos \psi
$$

where $R_{i}$ is the distance from the c.o.m. to the rotation axis, $\theta$ is the angle that $R_{i}$ forms with the vertical plane, and $\psi$ is the inclination angle of the rotation axis relative to the horizontal plane.

The ESM is a more efficient static stability measurement. It gives a qualitative idea of the amount of impact energy the vehicle withstands and also considers the height of the c.o.m. However, the ESM still does not consider any dynamic effects that might disturb vehicle stability. The ESM considers neither the effect of compliant terrains nor the stabilizing effect of a non-supporting leg. This is precisely what was proposed by Nagy 28 as an extension of the ESM that considers foot sinkage on soft and compliant terrain (the Compliant Energy Stability Margin, CESM), and Nagy also extended the concept to consider the stabilizing effect of a leg of a foot that is in the air (the Tipover Energy Stability Margin, TESM). For most walking machines, the ESM and the TESM coincide, because the non-supporting legs are too far from the floor to enhance stability. Only frame-based vehicles will find this stability margin an advantage.

Finally, Hirose et al. normalized the ESM to the robot weight and proposed the Normalized Energy Stability Margin, NESM, defined as 29 :

$$
S_{N E S M}=\frac{S_{E S M}}{m g}=\min _{i}^{n_{s}}\left(h_{i}\right)
$$


The NESM was shown to be the most efficient stability margin for statically stable walking machines. However, when dynamic effects arise during walking, machine stability cannot be judged precisely. Such situations exist in real walking robot applications, and therefore dynamic stability margins are more suitable.

\subsection{Dynamic Stability Margins}

The first dynamic stability criterion for quadrupeds using crawl gaits was proposed by Orin as an extension of the Center of Gravity Projection Method. The Center of Pressure Method, COP ${ }^{30}$, claims that a robot is dynamically stable if the projection of the c.o.m. along the direction of the resultant force acting on the c.o.m. lies inside the support polygon. The Dynamic Stability Margin is thus defined as the smallest distance from the COP to the edges of the support polygon (see also ref. ${ }^{16}$ ). The COP Method coincides with the Center of Gravity Projection method under static conditions and uneven terrain. Thus, it presents the same limitations.

Kang et al. lately renamed the COP the Effective Mass Center, EMC ${ }^{17}$, and redefined it as the point on the support plane where the resultant moment due to terrain-reaction forces and moments vanishes. In the literature of biped robots, this point is commonly named as the Zero Moment Point, ZMP 13, 31. However, Yoneda and Hirose stated that the CME or ZMP method is not valid for uneven terrain because the support polygon is not confined in a plane 20 .

Some momentum-based stability criteria have been defined as well. Here only the most meaningful ones are reviewed. The statement is as follows: Given a robot + manipulator system as shown in Figure 2(a), the forces and moments acting on the c.o.m. may destabilize it, making the system tumble. Dynamic equilibrium at the c.o.m. requires:

$$
\begin{aligned}
& \mathrm{F}_{\mathrm{I}}=\mathrm{F}_{\mathrm{S}}+\mathrm{F}_{\mathrm{G}}+\mathrm{F}_{\mathrm{M}} \\
& \mathrm{M}_{\mathbf{I}}=\mathrm{M}_{\mathrm{S}}+\mathrm{M}_{\mathrm{G}}+\mathrm{M}_{\mathrm{M}}
\end{aligned}
$$

where subscripts I, S, G and M denote inertia, support, gravitational and manipulation effects, respectively. 


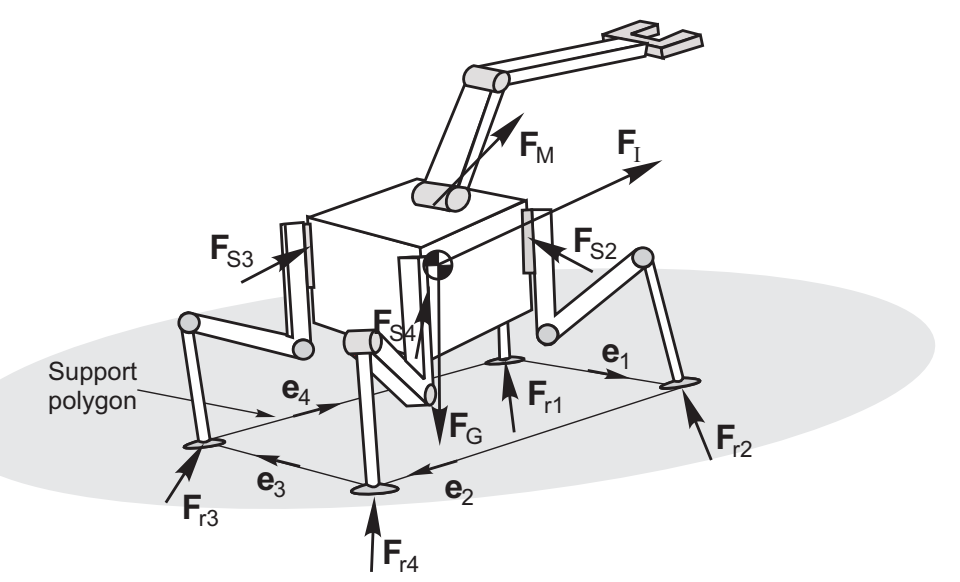

(a)

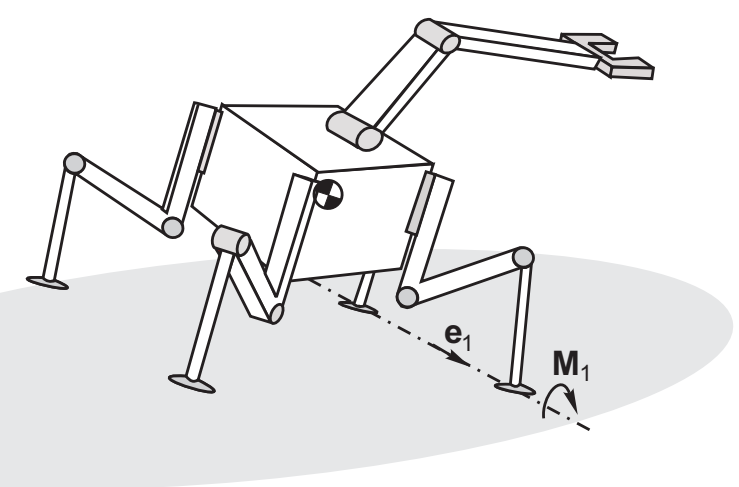

(b)

Figure 2: (a) Forces acting on a robot + manipulator system. (b) Robot tumbling around the rotation axis.

During the tumble the robot loses most of its support feet, leaving only those that conform a rotation axis (see Figure 2(b)). An interaction force $F_{R}$ and moment $M_{R}$ between robot and terrain result from the addition of reaction forces at every foot $\left(F_{r_{i}}\right)$ and the momentum they generate around the c.o.m., respectively. This reaction force and moment generate a moment $M_{i}$ about the rotation axis $i$ that must compensate for the destabilizing forces and moments to ensure system stability. When such compensation is not enough, the system is said to be dynamically unstable.

Based on this statement, Lin and Song 18 redefined the Dynamic Stability Margin, DSM, as the smallest of all moments $M_{i}$ for every rotation axis in the support polygon, 
normalized by the weight of the system, that is:

$$
S_{D S M}=\min _{i}\left(\frac{M_{i}}{m g}\right)=\min _{i}\left(\frac{\mathbf{e}_{i} \cdot\left(\mathbf{F}_{R} \times \mathbf{P}_{i}+\mathbf{M}_{R}\right)}{m g}\right)
$$

where $P_{i}$ is the position vector from the c.o.m. to the $i$-th support foot, and $\mathbf{e}_{\mathbf{i}}$ is a unit vector that goes round the support polygon in the clockwise sense, as shown in Figure 2(a). If all moments are positive (if they have the same direction and sense than $e_{i}$ ), then the system is stable.

Note that the term DSM is used for both Orin's dynamic stability margin and Lin and Song's criterion, but in this paper, the term DSM will be reserved for Lin and Song's criterion, while Orin's dynamic stability margin will be referred to as the EMC.

A few years later, Yoneda and Hirose 20 proposed the Tumble Stability Judgment, TSJ, based on the same statement. In the dynamic equilibrium of the system, they assumed massless legs, so leg-support and foot-reaction forces coincide. Therefore they obtained the resultant reaction force $F_{R}$ and moment $M_{R}$ from:

$$
\begin{aligned}
& \mathrm{F}_{\mathbf{R}}=\mathrm{F}_{\mathbf{I}}-\mathrm{F}_{\mathrm{G}}-\mathrm{F}_{\mathrm{M}} \\
& \mathrm{M}_{\mathbf{R}}=\mathrm{M}_{\mathbf{I}}-\mathrm{M}_{\mathrm{G}}-\mathrm{M}_{\mathrm{M}}
\end{aligned}
$$

Thus, the moment $M_{i}$ around the rotation axis is calculated as follows:

$$
M_{i}=\mathbf{M}_{\mathbf{R}} \cdot \mathbf{e}_{\mathbf{i}}+\mathbf{F}_{\mathbf{R}} \times \mathbf{p}_{\mathbf{i}} \cdot \mathbf{e}_{\mathbf{i}}
$$

Note that the moment calculated by (9) is exactly the same as is used in (6).

The TSJ states that the system is dynamically stable if there exists any support foot $j$ in the direction of rotation that prevents the system from tumbling. Then, the Tumble Stability Margin, TSM, becomes:

$$
S_{T S M}=\min _{i}\left(\frac{M_{i}}{m g}\right)
$$

Recently Zhou et al. ${ }^{32}$ proposed the Leg-end Supporting Moment criterion, LSM. This stability margin is exactly the same as the TSM, but its users obtain the resultant 


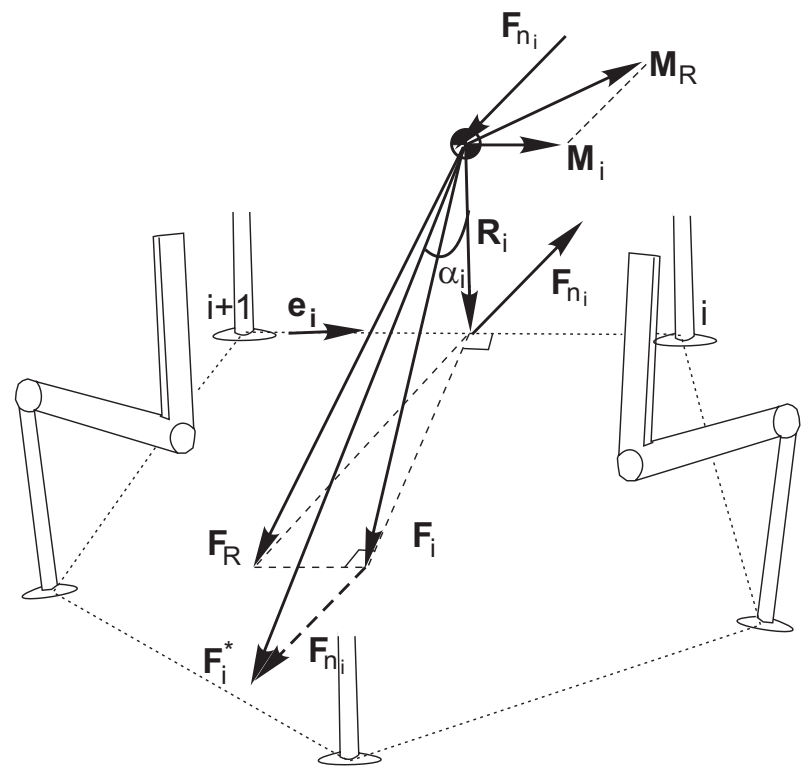

Figure 3: Geometric problem of the Force-Angle stability margin.

force $F_{R}$ and moment $M_{R}$ from force sensors at the feet. Therefore, the LSM stability margin avoids the sort of errors that can appear in the TSM from neglecting leg dynamics.

Apart from ZMP-based and momentum-based stability criteria, a different criterion was proposed by Papadopoulos and Rey ${ }^{19}$. The Force-Angle stability criterion finds the angle $\alpha_{i}$ between the resultant force acting from the c.o.m. on the ground (the opposite to the reaction force $F_{R}$ ) and the vector $R_{i}$, normal to the rotation axis from the c.o.m. (see Figure 3). The system becomes unstable when this angle becomes zero. The stability margin is the product of the angle times the resultant force $F_{R}$, that is:

$$
S_{F A S M}=\min \left(\alpha_{i}\right) \cdot\left\|\mathbf{F}_{R}\right\|
$$

These are the main stability criteria used today in walking machine control. Some of them seem to define the same stability margins, and there are no clear differences between them. Furthermore, their suitability for judging stability in any real situation, e. g. on sloped terrain, or where there are manipulation forces and moments or dynamic effects during the transfer of the legs, cannot be extracted directly from the definitions.

The following sections of this paper are devoted to coping with this lack of qualitative 
information about existing stability margins. For this purpose, a comparative analysis of stability margins is presented for different static and dynamic situations. This analysis was carried out through simulation of a walking robot on different terrain profiles and in different dynamic situations. The following section resumes the main features of the simulation, and Section 4 describes the comparative analysis and simulation results.

\section{SIMULATION SETUP}

To set up the features of the stability margins reviewed herein above, a comparative analysis was performed through simulation. A commercial Simulation Construction Set (SCS) 33 was chosen for this purpose, because it provides suitable tools for dynamic simulation. The SILO4 quadruped robot was used as a comparative testbed 34 , and the stability margins were computed while the robot was walking using a two-phase discontinuous gait 35 . Using the Java-based SCS library, robot kinematics and dynamics were defined, as well as the ground profile and ground contact model. The simulation parameters and main features are described herein below.

\subsection{Simulation Parameters}

The integrator used for the simulation is based on the Runge-Kutta 4th-order method with an integration period of $0.4 \mathrm{~ms}$. However, the data is collected for graphic comparison at a sampling time of 0.02 seconds.

\subsection{Kinematic and Dynamic Modeling}

Robot kinematics are defined as a tree of joints. Therefore, the body is the root joint, and four branches of joints define the four legs. Each leg consists of three pin joints, and each joint has a link associated to it, which defines the shape, size and color of the structure.

The mass and inertia of each link are also defined so that the robot's dynamics can be computed from the defined parameters using the Featherstone algorithm 36 . 


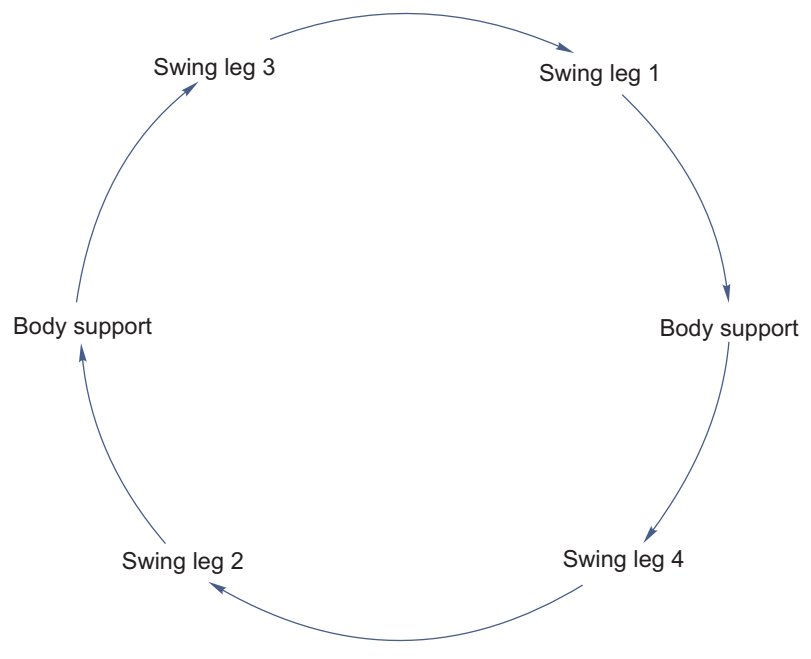

Figure 4: Finite state machine for a two-phase discontinuous gait.

\subsection{Gait Control}

The SILO4 robot walks using a two-phase discontinuous gait, which is programmed by means of a finite state machine, shown in Figure 4. Two swing states precede each body support phase, where the body is propelled forward. The swing of a leg consists of three straight-line trajectories at the foot (lift, forward motion and landing), which are generated on-line. The body support phase consists of the straight backward motion of the four legs simultaneously. Every trajectory generation process determines desired joint trajectories, which are PD-controlled at the joint level, that is:

$$
\tau_{i}=K_{p}\left(\theta_{i}^{\text {des }}-\theta_{i}\right)+K_{v}\left(\dot{\theta}_{i}^{\text {des }}-\dot{\theta}_{i}\right)
$$

where subscript $i$ denotes the joint number, $\theta$ and $\dot{\theta}$ are joint position and velocity, respectively, $\theta^{d e s}$ and $\dot{\theta}^{\text {des }}$ are the reference joint position and velocity, respectively, and $K_{p}$ and $K_{v}$ are the elastic and dumping constants. The output of the PD controller in (12) is the torque $\tau_{i}$ required at the joint $i$. 


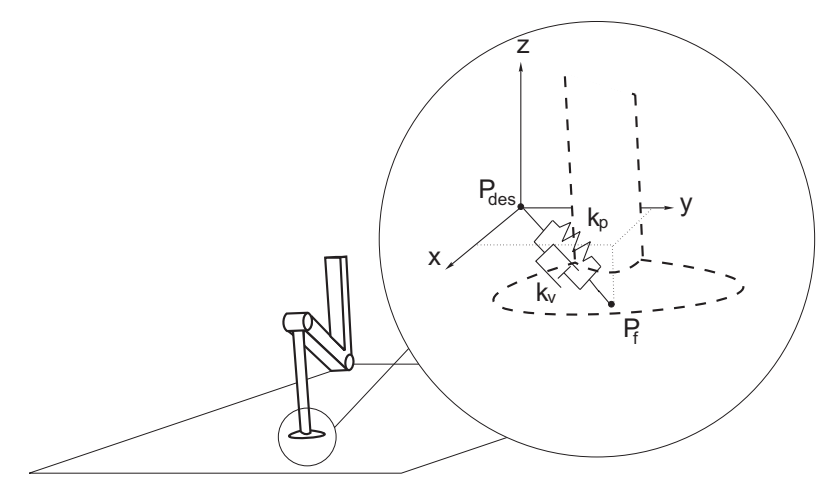

Figure 5: Ground contact model

\subsection{Ground Profile}

The shape of the terrain is programmed as an elevation function relative to a fixed reference frame. Therefore a sloped terrain in the $x$ direction is described by the following function:

$$
z=\alpha x
$$

where $z$ is the height of the terrain surface, and $\alpha$ represents the slope.

A random uneven terrain can be modeled by:

$$
z=A_{1} \sin \left(\omega_{1} x+\varphi_{1}\right) A_{2} \sin \left(\omega_{2} y+\varphi_{2}\right)
$$

where $A_{1}$ and $A_{2}$ are the amplitude of roughness, $\omega_{1}$ and $\omega_{2}$ are their frequencies, and $\varphi_{1}$ and $\varphi_{2}$ denote their phases.

\subsection{Ground Contact Model}

The dynamic model of the robot/terrain interaction consists of three orthogonal springdumper systems along the simulated $x, y$ and $z$ spatial directions, respectively, attached to the feet (see Figure 5). Each time the $z$ coordinate of a foot enters the ground profile, a ground-reaction force is applied against it, whose Cartesian coordinates are given by:

$$
F_{x}=k_{p}\left(x_{d e s}-x_{f}\right)-k_{v} \dot{x}_{f}
$$




$$
\begin{aligned}
& F_{y}=k_{p}\left(y_{d e s}-y_{f}\right)-k_{v} \dot{y}_{f} \\
& F_{z}=k_{p}\left(z_{\text {des }}-z_{f}\right)-k_{v} \dot{z}_{f}
\end{aligned}
$$

where $\left(x_{d e s}, y_{d e s}, z_{d e s}\right)$ are the cartesian coordinates of the point $P_{\text {des }}$ at the initial foot/terrain contact, and $\left(x_{f}, y_{f}, z_{f}\right)$ are the coordinates of point $P_{f}$, which represents the foot position at any later instant.

Joint elasticity can be also modeled using the ground contact model. Assuming a Cartesian spring-dumper model of joint compliance 37 , the composition of the elastic and dumping effects of the three joints of a leg at the instant that the foot contacts the ground can be considered an equivalent spring-dumper system at the foot. Therefore, the addition of the equivalent elastic and dumping constants to the ground contact model reflects the additional effect of joint elasticity during walking.

Once the simulation features are defined, the different stability margins for walking machines can be analyzed and compared during simulation. The next section describes this analysis and shows some simulation results.

\section{A COMPARATIVE STUDY OF STABILITY MARGINS}

The goal of this comparative study is to produce a qualitative classification of stability margins to determine which is most suitable for each given application. The stability margins that have been selected for the analysis are SSM, NESM, DSM, TSM, FASM and EMC. They have been computed while the robot was walking using a two-phase discontinuous gait in the following six different terrain and dynamic situations:

CASE 1: Horizontal, even terrain in the absence of dynamics.

CASE 2: Uneven terrain in the absence of dynamics.

CASE 3: Horizontal, even terrain when inertial and elastic effects arise. 


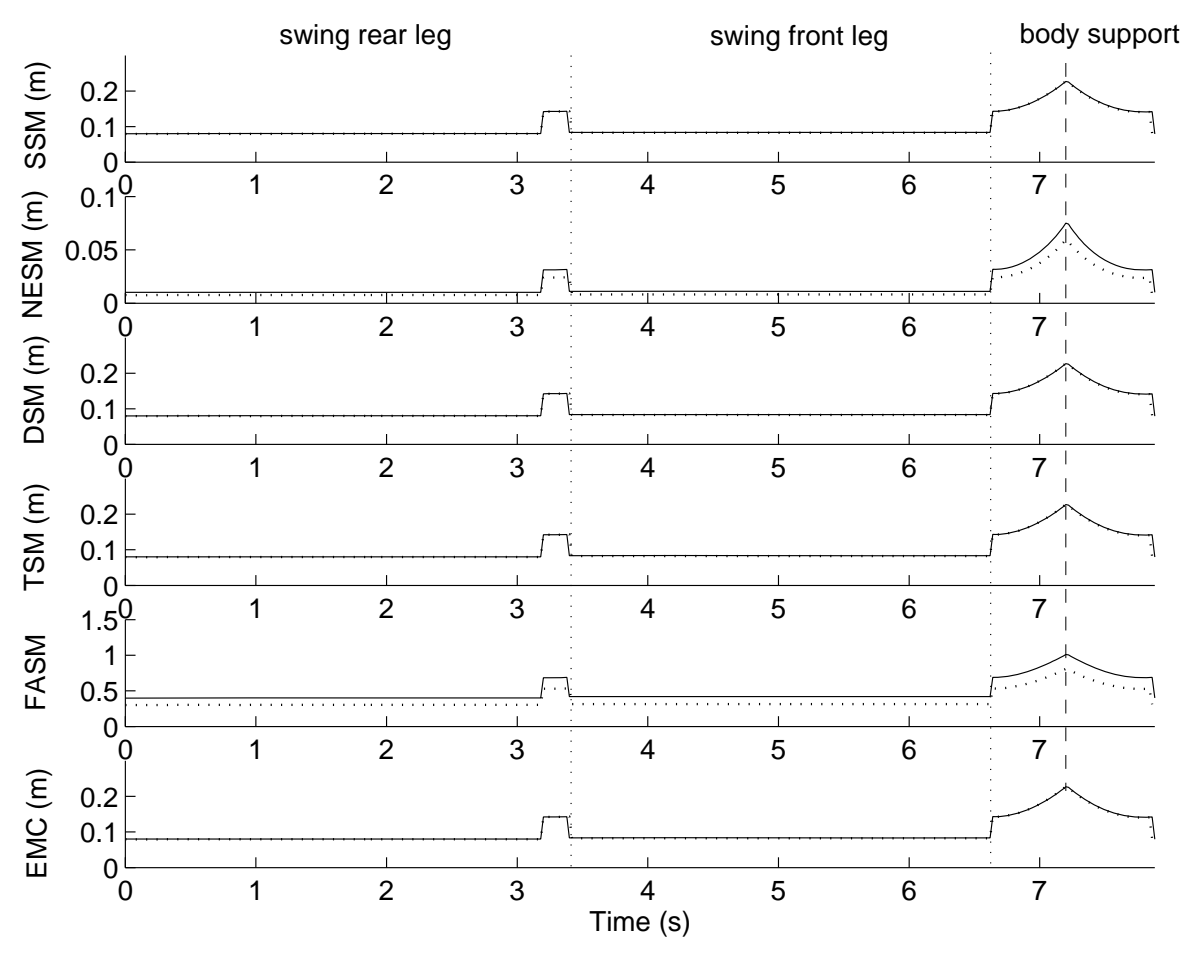

Figure 6: Different stability margins in the absence of system dynamics on horizontal terrain (CASE 1). Solid line for a robot height of $320 \mathrm{~mm}$ and dotted line for a robot height of $420 \mathrm{~mm}$.

CASE 4: Uneven terrain when inertial and elastic effects arise.

CASE 5: Horizontal, even terrain when inertial, elastic and manipulation dynamics arise.

CASE 6: Uneven terrain when inertial, elastic and manipulation dynamics arise.

The above six case studies represent different situations that a robot can find in real applications.

Figures 6 and 7 show one half of the gait cycle for cases 1 and 2, respectively. The gait phases corresponding to the swing of the rear and front legs precede the body support phase. From both figures it can be observed that the SSM, DSM, TSM and EMC coincide. The margins also coincide for different heights of the c.o.m. (dotted line). It is relevant that in the first case study (see Figure 6), when the terrain is horizontal and even, these four margins do not vary with c.o.m. height. This is a drawback of all four criteria 


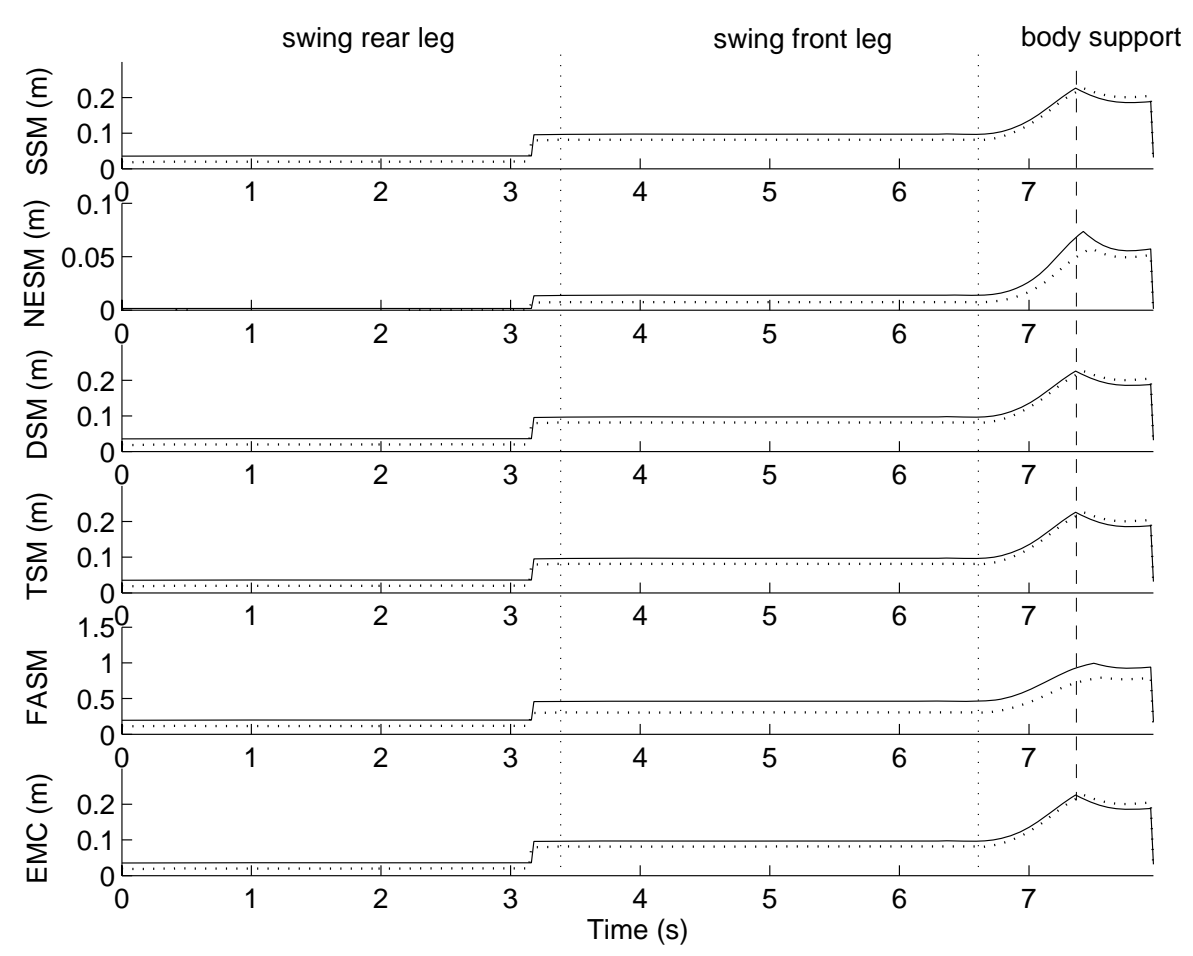

Figure 7: Different stability margins in the absence of system dynamics on terrain inclined $10^{\circ}$ from the horizontal plane (CASE 2). Solid line for a robot height of $320 \mathrm{~mm}$ and dotted line for a robot height of $420 \mathrm{~mm}$.

because, obviously, the increase of the c.o.m. height has a destabilizing effect. However, in the second case study, for uneven terrain (see Figure 7), all six margins consider c.o.m. height. The vertical dashed line inside the body-support-phase interval points to the instant when the SSM is maximum; on horizontal terrain this instant is one half of the support-phase interval. For both case studies that instant coincides for all margins.

The NESM and FASM are the only margins that reflect the effect of body height increase on horizontal and even terrain. Thus, they are the only margins that give a successful stability measurement.

On an inclined surface (see Figure 7), the NESM and FASM differ from the others in their instant of maximum stability. The NESM reaches maximum after the SSM, DSM, TSM and EMC (which coincide). Also the maximum FASM occurs even later than the maximum NESM. The main question at this point seems to be: Which of the margins is 


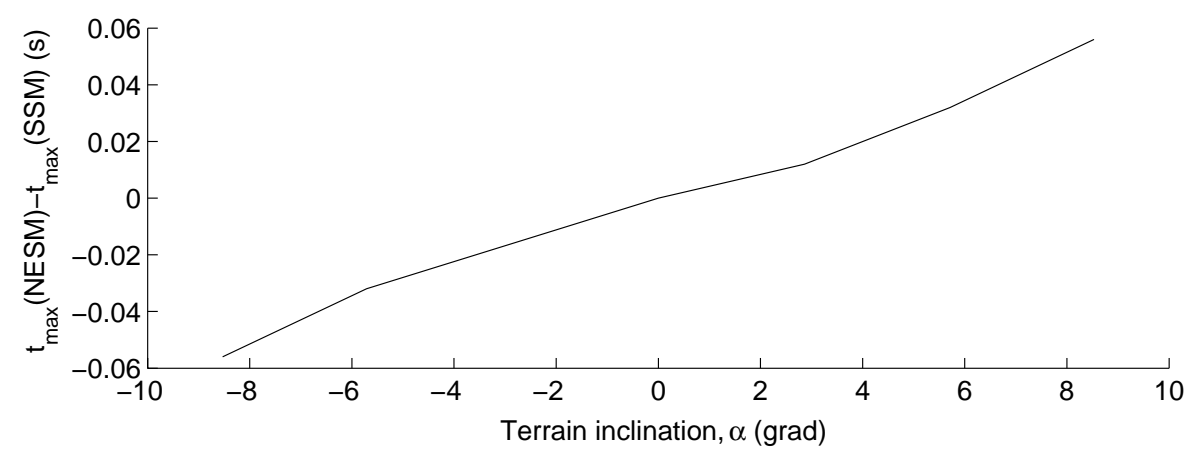

(a)

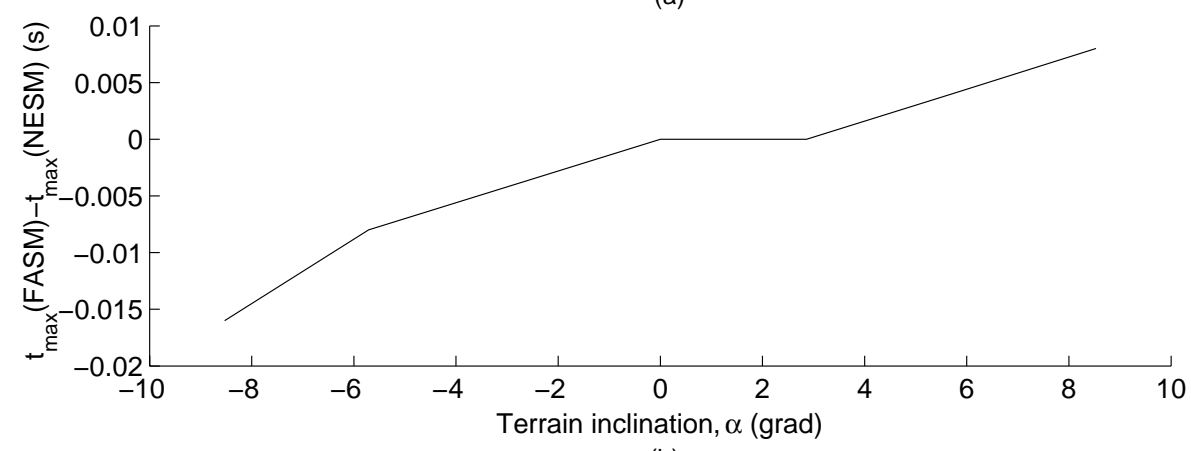

(b)

Figure 8: Difference between instants of maximum stability for several terrain inclinations. (a) NESM and SSM. (b) FASM and NESM.

the best? The NESM is defined as the measurement of impact energy that the system can absorb during the tumble. Thus, when the c.o.m. is placed at the maximum NESM point, the possibility of tumbling downhill is equal to uphill (see also ${ }^{20}$ ). Therefore, the NESM is the optimum margin.

Figure 8 (a) shows the difference between the instants of maximum NESM and SSM as a function of the terrain inclination angle, while Figure $8(\mathrm{~b})$ shows the difference between the instants of maximum FASM and NESM. Figure 8(a) demonstrates that the instant of maximum SSM always precedes the instant of maximum NESM for different positive and negative terrain inclinations. Furthermore, the instant of maximum FASM always follows the instant of maximum NESM, as shown in Figure 8(b) for different terrain inclinations. Therefore, the instants of maximum SSM and FASM only coincide with the instant of optimum stability when the terrain is horizontal and even. If there is a slope in the terrain, the SSM and FASM will never be optimal. 


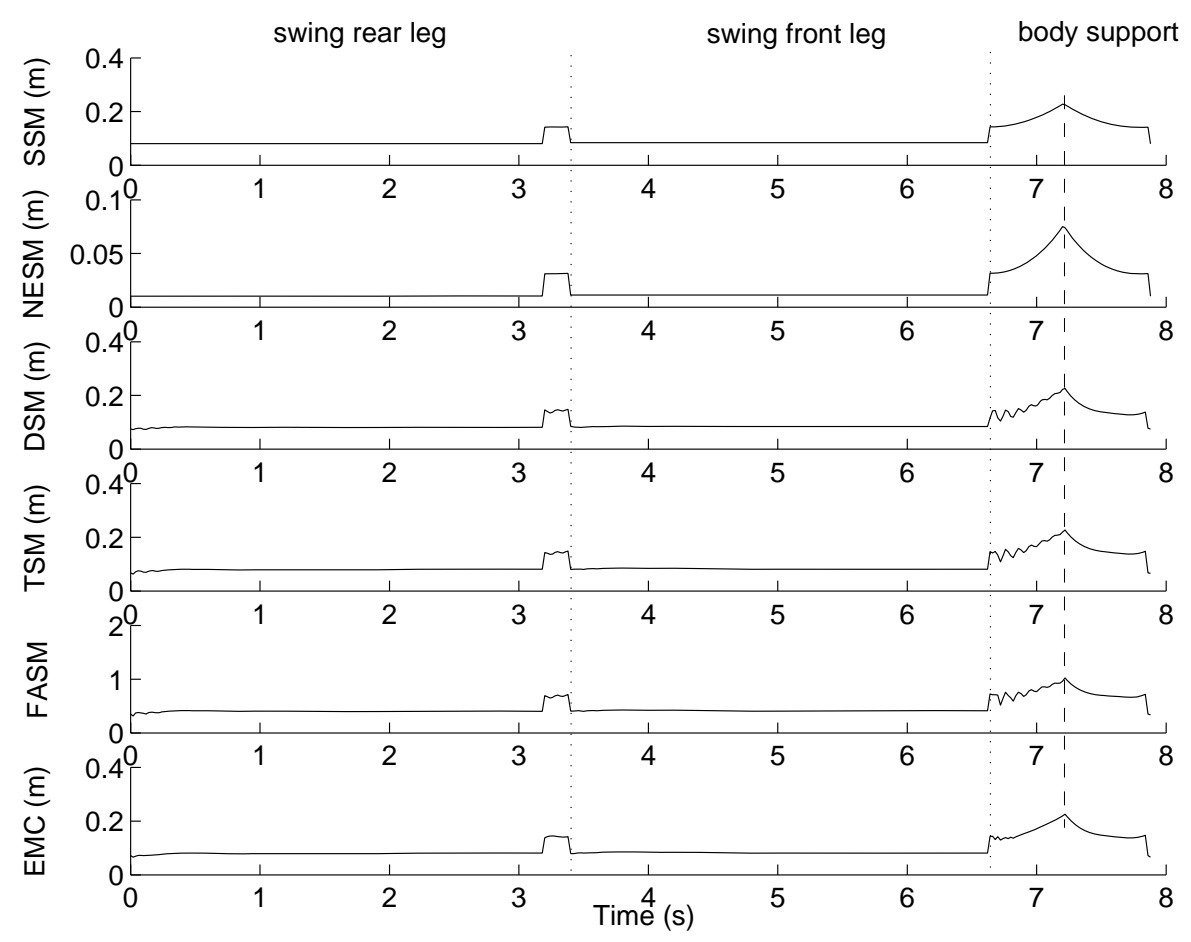

Figure 9: Different stability margins when inertial and elastic effects arise on horizontal terrain (CASE $3)$.

Figures 9 and 10 show one half of the gait cycle for cases 3 and 4 respectively, which correspond to the existence of inertial effects when the robot is walking over horizontal and inclined terrain, respectively. Elastic effects due to joint elasticity and ground contact effects are introduced as well.

On horizontal terrain (see Figure 9), all the instants of maximum stability still coincide. However, the DSM, TSM, FASM and EMC reflect some oscillation of the margin due to joint elasticity at leg lift, placement and body support. Inertial effects during the leg transfer phase and body support are reflected as well. These dynamic effects are not reflected by the SSM and NESM, because they are static stability margins only. Figure 11 shows this difference, plotting a comparison between the SSM and the DSM. The DSM undergoes a decrease in stability due to inertial effects at leg lift and body propulsion. Vibrations due to joint elasticity are also reflected at leg lift, foot placement and body support. 


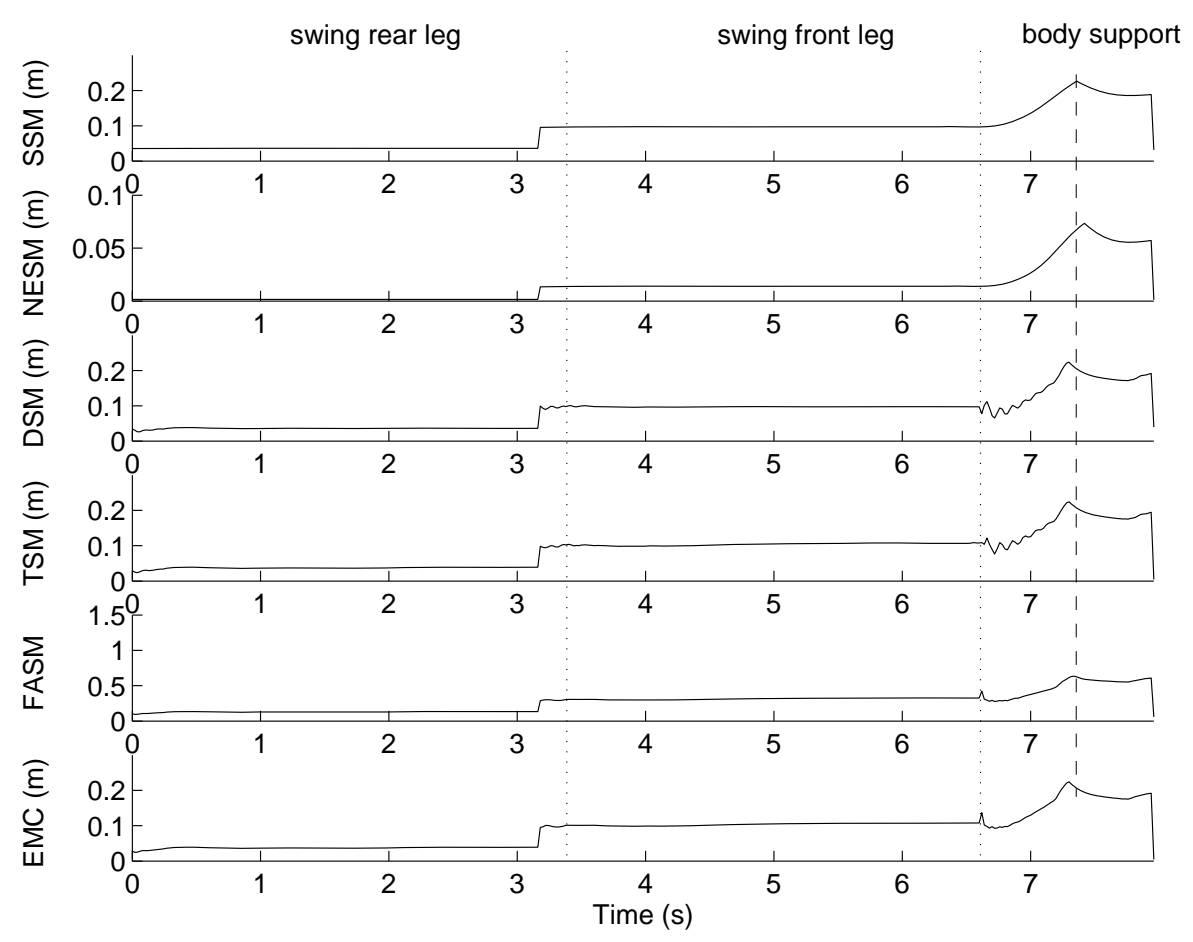

Figure 10: Different stability margins when inertial and elastic effects arise on terrain inclined $10^{\circ}$ from the horizontal plane (CASE 4).

Therefore, only dynamic stability criteria are valid for judging stability when inertial and elastic effects are involved. However, the DSM, TSM and EMC have the same failing here as on horizontal terrain: They do not consider the effect of height changes. Only the FASM is suitable for case study 3. Nevertheless, if robot height is not presumed to change, the DSM, TSM and EMC are suitable also.

Figure 10 depicts stability margins for the fourth case study, when the terrain is inclined and inertial and elastic effects arise. While the SSM and NESM do not reflect any reduction of the stability margin due to dynamics (as they are the same as in Figure 7), the DSM, TSM, FASM and EMC reflect a decrease in stability. Also their instants of maximum stability occur some time earlier than in case study 2 , where there were dynamic effects. Figure 12 explains this fact. It depicts a planar robot and its two support feet 1 and 2 during the body-support phase. The c.o.m. moves from position $G_{1}$ to $G_{4}$. At $G_{2}$, the c.o.m. is at one half of the support phase. At this moment the velocity of the c.o.m. 


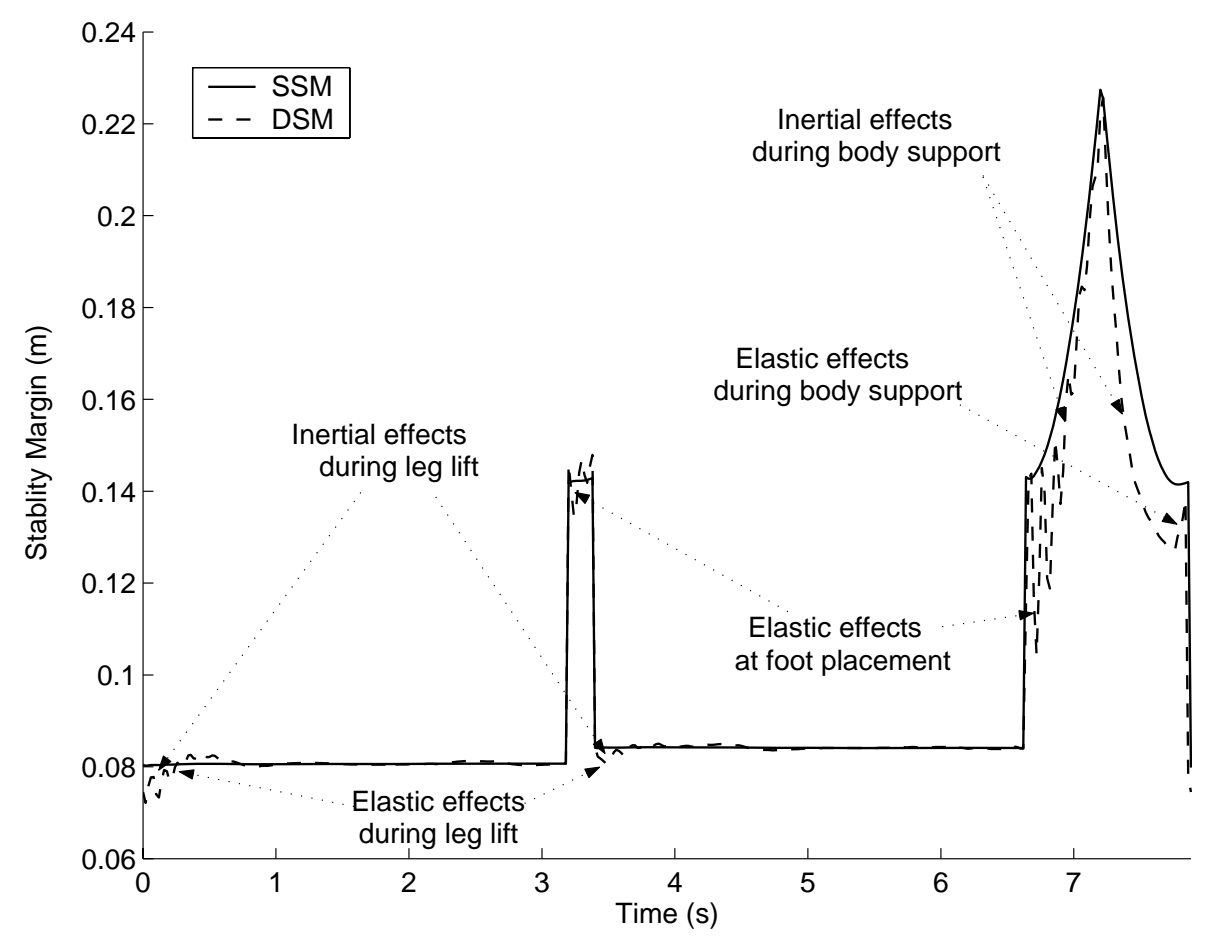

Figure 11: SSM and DSM during half gait cycle on horizontal terrain when inertial and elastic effects arise.

is maximum, and the acceleration is zero. Thus, there are no inertial forces, and gravity is the only resultant force. Some time later, at $G_{3}$, the body is decelerating, and the resultant force makes the DSM maximum. Finally, at $G_{4}$ the SSM is maximum. Points $G_{3}$ and $G_{4}$ only coincide if the resultant force at $G_{4}$ is vertical (gravity). That only occurs in the case of horizontal terrain, and then $G_{2}, G_{3}$ and $G_{4}$ meet, as in Figure 9.

The maximum stability instant of the FASM occurs later than that of the DSM, TSM and EMC. However, the optimum criterion is the one whose maximum stability instant takes place before the FASM and after the DSM, TSM and EMC. Therefore, no optimum stability criterion exists for case study 4.

Figures 13 and 14 show one half of the gait cycle for cases 5 and 6 , respectively, which correspond to the existence of manipulation effects when the robot walks over horizontal and inclined terrain, respectively. Inertial and elastic effects are considered as well. Both figures show that manipulation forces opposing motion cause a stability decrease at the 


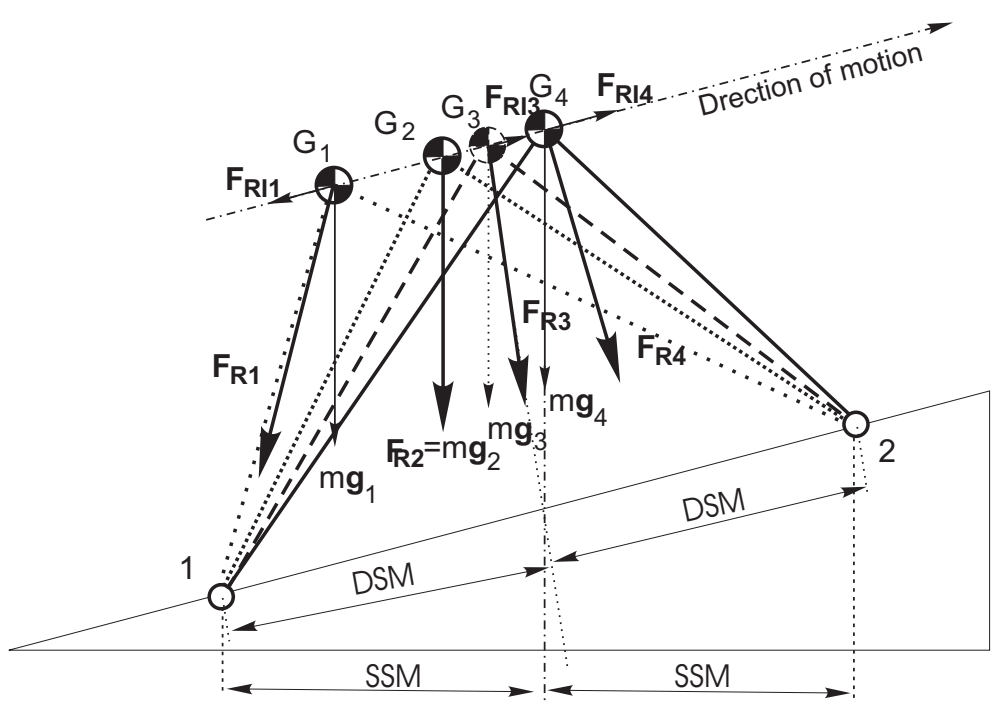

Figure 12: Different c.o.m. positions during the body support phase.

rear leg's swing phase and an increase at the front leg's swing phase. Also, a delay of the maximum stability instant can be observed in the DSM, TSM, FASM and EMC. It is obvious from the figure that if the manipulation force is increased the robot could be destabilized during the swing of the rear leg. This will never be foreseen by the SSM and NESM.

The instant of maximum FASM takes place after the instant of maximum DSM, TSM and EMC. However, the optimum criterion should meet the maximum stability position after the DSM and before the FASM. This is shown in Figures 15(a) and (b), where the instants of maximum DSM and FASM are compared with an energy-based optimum criterion for different terrain inclination angles and different manipulation forces. As both figures show, neither the DSM nor the FASM coincide with the optimum criterion. Thus, no optimum criteria exists for the fifth and sixth case studies.

Table 1 summarizes a classification of the stability margins studied herein. The symbol " $\sqrt{ }$ " denotes that the criterion is "valid," the symbol " $\times$ " denotes "not valid," and the symbol "*" denotes "optimum." Only the NESM provides the optimal measurement as static stability margin. However all of them are valid. As dynamic stability margins the SSM and NESM are not valid. When inertial effects arise over horizontal terrain, 


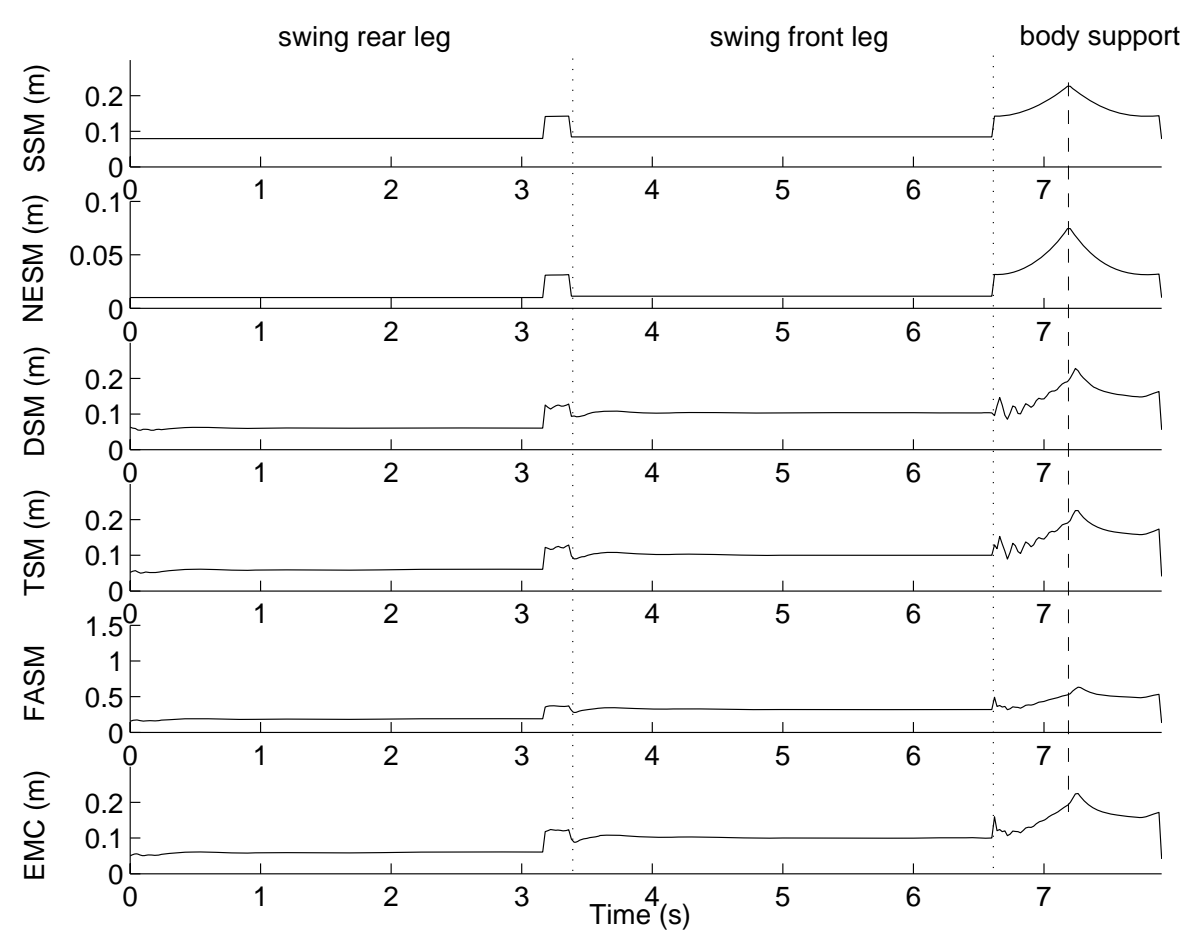

Figure 13: Different stability margins when inertial, elastic and manipulation effects arise as a 20-N constant force opposing motion on horizontal terrain (CASE 5).

only the FASM provides the optimum measurement, yet the rest of the dynamic stability criteria are valid. When any other dynamic effects are present, such as manipulation forces and moments, over horizontal or uneven terrain, there is no criterion that provides the optimum margin. Therefore there is no criterion that can assure the stability of a machine under those conditions. Another conclusion of the study is that the DSM, TSM and EMC yield the same measurement for every situation studied herein.

The last comparison of the selected criteria according to their computational complexity was obtained by finding the number of mathematical operations required for simulation. Table 2 shows these data, represented as the number of additions, multiplications, trigonometric operations and square roots computed in each simulation step, considering a support polygon of $n$ sides. In this calculation, foot reaction forces are assumed to be known. As the table shows, the FASM is the most complex of the compared margins, while the DSM is the least complex of the dynamic criteria and the 


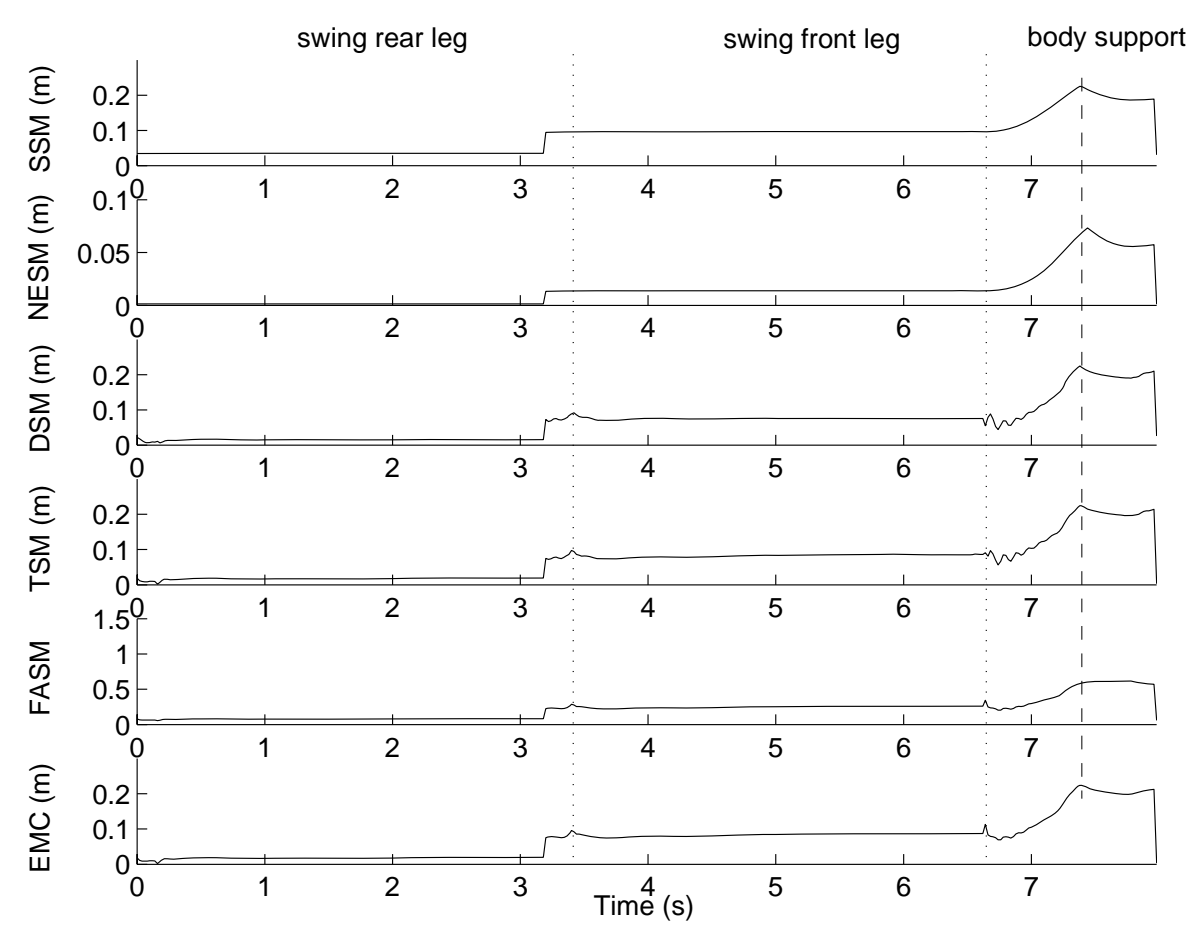

Figure 14: Different stability margins when inertial, elastic and manipulation effects arise as a 20-N constant force opposing motion on terrain inclined $10^{\circ}$ from the horizontal plane (CASE 6).

SSM is the least complex of the static criteria.

\section{CONCLUSIONS}

Several stability margins have been defined in the course of research on walking robots, yet none of their definitions directly suggest anything about their suitability to judge stability in any real situation, e. g. on sloped terrain, or in the presence of manipulation forces and moments or dynamic effects during leg transfer.

This paper has been devoted to coping with this lack of qualitative information about existing stability margins. For this purpose, a comparative study has been run on stability margins in different static and dynamic situations. This analysis has been carried out through simulation of a walking robot using a two-phase discontinuous gait in six different case studies where various terrain profiles and dynamic situations have been considered. 
Table 1: Classification of existing stability criteria.

\begin{tabular}{ccccccccc}
\hline $\begin{array}{c}\text { Uneven } \\
\text { terrain }\end{array}$ & $\begin{array}{c}\text { Inertial } \\
\text { effects }\end{array}$ & $\begin{array}{c}\text { Manipulation } \\
\text { forces }\end{array}$ & SSM & NESM & DSM & TSM & FASM & EMC \\
\hline no & no & no & $\sqrt{ }$ & $*$ & $\sqrt{ }$ & $\sqrt{ }$ & $*$ & $\sqrt{ }$ \\
no & yes & no & $\times$ & $\times$ & $\sqrt{ }$ & $\sqrt{ }$ & $*$ & $\sqrt{ }$ \\
no & yes & yes & $\times$ & $\times$ & $\sqrt{ }$ & $\sqrt{ }$ & $\sqrt{ }$ & $\sqrt{ }$ \\
yes & no & no & $\sqrt{ }$ & $*$ & $\sqrt{ }$ & $\sqrt{ }$ & $\sqrt{ }$ & $\sqrt{ }$ \\
yes & yes & no & $\times$ & $\times$ & $\sqrt{ }$ & $\sqrt{ }$ & $\sqrt{ }$ & $\sqrt{ }$ \\
yes & yes & yes & $\times$ & $\times$ & $\sqrt{ }$ & $\sqrt{ }$ & $\sqrt{ }$ & $\sqrt{ }$ \\
\hline
\end{tabular}

Table 2: Computational complexity of existing stability criteria.

\begin{tabular}{lcccccc}
\hline & SSM & NESM & DSM & TSM & FASM & EMC \\
\hline Additions & $17 \mathrm{n}$ & $33 \mathrm{n}$ & $44 \mathrm{n}$ & $86 \mathrm{n}$ & $109 \mathrm{n}$ & $67 \mathrm{n}$ \\
Products & $13 \mathrm{n}$ & $23 \mathrm{n}$ & $39 \mathrm{n}$ & $90 \mathrm{n}$ & $117 \mathrm{n}$ & $70 \mathrm{n}$ \\
Trigonometric & - & - & - & - & $3 \mathrm{n}$ & - \\
Square roots & $\mathrm{n}$ & $2 \mathrm{n}$ & $2 \mathrm{n}$ & $3 \mathrm{n}$ & $6 \mathrm{n}$ & $2 \mathrm{n}$ \\
\hline
\end{tabular}

These case studies cover all the situations that can occur during real industrial applications of legged robots.

As a result, a classification of stability criteria has been proposed showing that no optimum criterion exists for every situation studied. Also, it has been shown that every momentum-based stability criterion provides the same stability margin, and no criterion is ever optimal. The selected criteria have been also compared in terms of their computational complexity. This classification enables the proper stability criterion to be chosen for each real application.

\section{Acknowledgements}

The authors want to thank Jerry Pratt for his help using the Yobotics! Simulation Construction Set software package.

This work has been partially funded by CICYT through Grant DPI2001-1595. The CAM sponsored a scholarship granted to the first author to perform this work. 


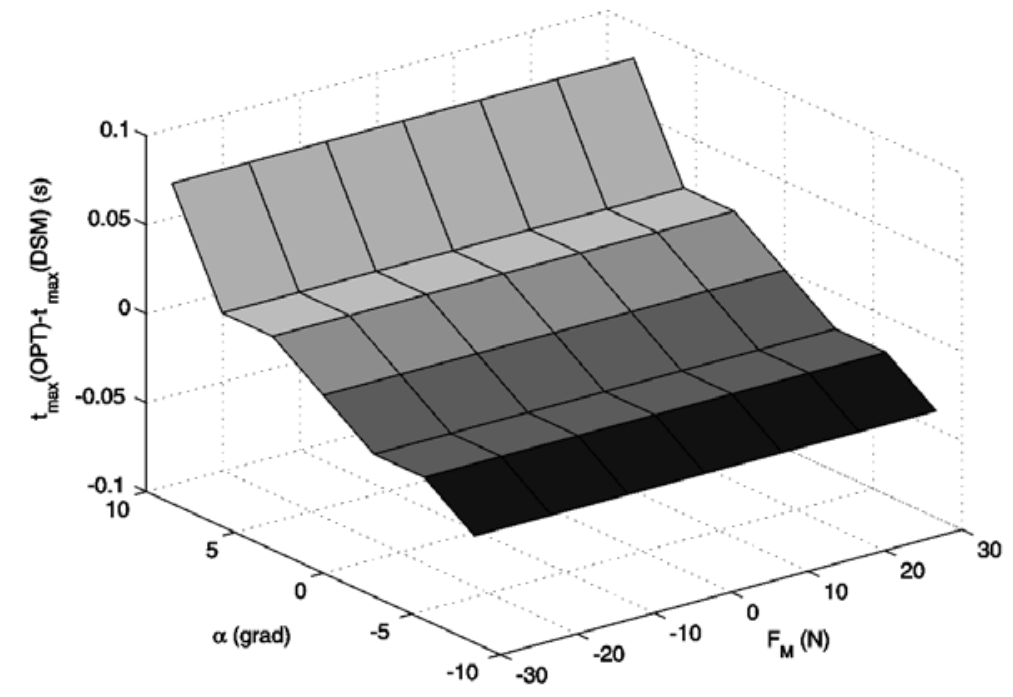

(a)

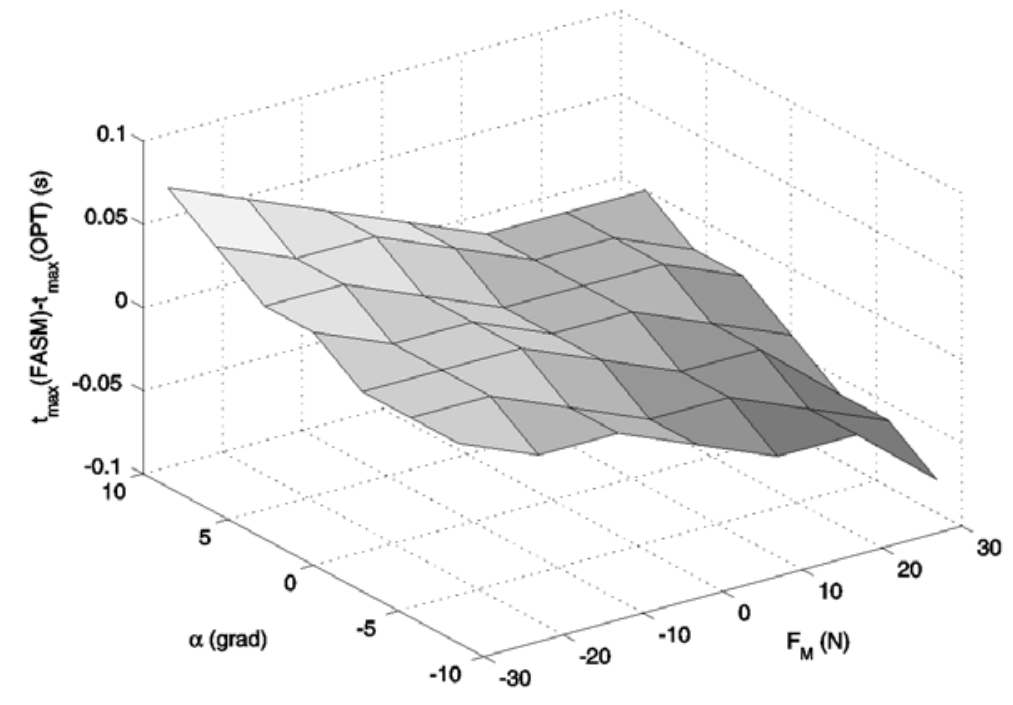

(b)

Figure 15: Difference between instants of maximum stability for several terrain inclinations $(\alpha)$ and manipulation forces $\left(F_{M}\right)$. (a) SSM and optimum criterion. (b) FASM and optimum criterion. 


\section{References}

[1] R. McGhee and A. Frank, "On the stability properties of quadruped creeping gaits," Mathematical Bioscience 3, 331-351, 1968.

[2] V. Kumar and K. Waldron, A review of research on walking vehicles, 243-266. In O. Khatib, J.J. Craig and T. Lozano-Perez, editors. The robotics review, The MIT Press, Cambridge, Massachusetts, 1989.

[3] S. Song and K. Waldron, Machines that walk: the adaptive suspension vehicle, The MIT Press, Cambridge, Massachusetts, 1989.

[4] S. Arimoto and F. Miyazaki, "Biped locomotion robots," JARECT Computer Science and Technologies 12, 194-205, 1984.

[5] Y. Fujimoto, S. Obata, and A. Kawamura, "Robust biped walking with active interaction control between foot and ground," in Proc. IEEE Int. Conf. Robotics and Automation, 2030-2035, May 1998. Leuven, Belgium.

[6] J. Furusho and A. Sano, "Sensor-based control of a nine-link biped," The International Journal of Robotic Research 9(2), 83-98, 1990.

[7] K. Hirai, M. Hirose, Y. Haikawa, and T. Takenaka, "The development of Honda humanoid robot," in Proc. IEEE Int. Conf. Robotics and Automation, 1321-1326, May 1998. Leuven, Belgium.

[8] K. Löffler, M. Gienger, and F. Pfeiffer, "A biped jogging robot - theory and realization," in Proc. Int. Conf. Climbing and Walking Robots, 51-58, October 2000. Madrid, Spain.

[9] M. H. Raibert, Legged robots that balance, The MIT Press, Cambridge, Massachusetts, 1986. 
[10] M. Buehler, R. Battaglia, A. Cocosco, G. Hawker, J. Sarkis, and K. Yamazaki, "Scout: A simple quadruped that walks, climbs and runs," in Proc. IEEE Int. Conf. Robotics and Automation, 1701-1712, May 1998. Leuven, Belgium.

[11] M. Raibert, M. Chepponis, and H. Brown Jr., "Running on four legs as though they were one," IEEE Journal of Robotics and Automation RA2(2), 70-82, 1986.

[12] H. Wong and D. Orin, "Dynamic control of a quadruped standing jump," in Proc. IEEE Int. Conf. Robotics and Automation, 346-351, 1993. Atlanta, Georgia.

[13] A. Goswami, "Postural stability of biped robots and the foot-rotation indicator (fri) point," The International Journal of Robotic Research 18(6), 523-533, 1999.

[14] H. Kimura, I. Shimoyama, and M. H., "Dynamics in the dynamic walk of a quadruped robot," Advanced Robotics 4(3), 283-301, 1990.

[15] K. Yoneda, H. Iiyama, and S. Hirose, "Intermitent trot gait of a quadruped walking machine dynamic stability control of an omnidirectional walk," in Proc. IEEE Int. Conf. Robotics and Automation, 3002-3007, 1996. Atlanta, Georgia.

[16] P. Gonzalez de Santos, M. Jimenez, and M. Armada, "Dynamic effects in statically stable walking machines," Journal of Intelligent and Robotic Systems 23(1), 71-85, 1998.

[17] D. Kang, Y. Lee, S. Lee, Y. Hong, and Z. Bien, "A study on an adaptive gait for a quadruped walking robot under external forces," in Proc. IEEE Int. Conf. Robotics and Automation, 2777-2782, April 1997. Albuquerque, New Mexico.

[18] B. Lin and S. Song, "Dynamic modeling, stability and energy efficiency of a quadrupedal walking machine," in Proc. IEEE Int. Conf. Robotics and Automation, 367-373, 1993. Atlanta, Georgia. 
[19] E. Papadopoulos and D. Rey, "A new measure of tipover stability margin for mobile manipulators," in Proc. IEEE Int. Conf. Robotics and Automation, 3111-3116, April 1996. Minneapolis, MN.

[20] K. Yoneda and S. Hirose, "Three-dimensional stability criterion of integrated locomotion and manipulation," Journal of Robotics and Mechatronics 9(4), 267-274, 1997.

[21] J. Bobrow, S. Dubowsky, and J. Gibson, "Time-optimal control of robotic manipulators along specified paths," The International Journal of Robotic Research 4(3), 3-17, 1985.

[22] E. Garcia and P. Gonzalez de Santos, "Using soft computing techniques for improving foot trajectories in walking machines," Journal of Robotic Systems 18(7), 343-356, 2001.

[23] R. McGhee and G. Iswandhi, "Adaptive locomotion for a multilegged robot over rough terrain," IEEE Trans. on Systems, Man, and Cybernetics SMC-9(4), 176$182,1979$.

[24] C. Zhang and S. Song, "Gaits and geometry of a walking chair for the disabled," Journal of Terramechanics 26(3/4), 211-233, 1989.

[25] C. Zhang and S. Song, "Stability analysis of wave-crab gaits of a quadruped," Journal of Robotic Systems 7(2), 243-276, 1990.

[26] S. Mahalingham, W. Whittaker, and M. Gaithersburg, "Terrain adaptive gaits for walkers with completely overlapping work spaces," in Robots 13, 1-14, May 1989.

[27] D. Messuri, Optimization of the locomotion of a legged vehicle with respect to maneuverability. PhD thesis, The Ohio State University, 1985.

[28] P. Nagy, An investigation of walker/terrain interaction. PhD thesis, Carnegie Mellon University, 1991. 
[29] S. Hirose, H. Tsukagoshi, and K. Yoneda, "Normalized energy stability margin: generalized stability criterion for walking vehicles," in Proc. Int. Conf. Climbing and Walking Robots, 71-76, November 1998. Brussels.

[30] D. Orin, Interactive control of a six-legged vehicle with optimization of both stability and energy. PhD thesis, The Ohio State University, 1976.

[31] M. Vukobratovic, A. Frank, and D. Juricic, "On the stability of byped locomotion," IEEE Transactions on Biomedical Engineering 17(1), 25-36, 1970.

[32] D. Zhou, K. Low, and T. Zielinska, "A stability analysis of walking robots based on leg-end supporting moments," in Proc. IEEE Int. Conf. Robotics and Automation, 2834-2839, 2000. San Francisco, CA.

[33] Yobotics Inc., Boston, MA., Yobotics! Simulation Construction Set: Users Guide, 2002. http://www.yobotics.com/.

[34] E. Garcia, J. Galvez, and P. Gonzalez de Santos, "A mathematical model for the realtime control of the SILO4 leg," in Proc. Int. Conf. Climbing and Walking Robots, 447-460, October 2000. Madrid, Spain.

[35] P. Gonzalez de Santos and M. Jimenez, "Generation of discontinuous gaits for quadruped walking machines," Journal of Robotic Systems 12(9), 599-611, 1995.

[36] R. Featherstone, Robot Dynamics Algorithms, Kluwer Academic Publishers, BostonDordrecht-Lancaster, 1987.

[37] L. Shih, A. Frank, and B. Ravani, "Dynamic simulation of legged machines using a compliant joint model," The International Journal of Robotic Research 6(4), 33-46, 1987. 


\section{List of Figures}

1 Support polygon and different static stability margins. . . . . . . . . . 4

2 (a) Forces acting on a robot + manipulator system. (b) Robot tumbling around the rotation axis. . . . . . . . . . . . 7

3 Geometric problem of the Force-Angle stability margin. . . . . . . . . . 9

4 Finite state machine for a two-phase discontinuous gait. . . . . . . . . . . 11

$5 \quad$ Ground contact model . . . . . . . . . . . . . . . . 12

6 Different stability margins in the absence of system dynamics on horizontal terrain (CASE 1). Solid line for a robot height of $320 \mathrm{~mm}$ and dotted line for a robot height of $420 \mathrm{~mm} \ldots \ldots \ldots \ldots \ldots$. . . . . . . . 14

7 Different stability margins in the absence of system dynamics on terrain inclined $10^{\circ}$ from the horizontal plane (CASE 2). Solid line for a robot height of $320 \mathrm{~mm}$ and dotted line for a robot height of $420 \mathrm{~mm}$. . . . . . 15

8 Difference between instants of maximum stability for several terrain inclinations. (a) NESM and SSM. (b) FASM and NESM. . . . . . . . . . 16

9 Different stability margins when inertial and elastic effects arise on horizontal terrain $(\mathrm{CASE} 3) \ldots \ldots \ldots \ldots \ldots$

10 Different stability margins when inertial and elastic effects arise on terrain inclined $10^{\circ}$ from the horizontal plane (CASE 4). . . . . . . . . . 18

11 SSM and DSM during half gait cycle on horizontal terrain when inertial and elastic effects arise. . . . . . . . . . . . . 19

12 Different c.o.m. positions during the body support phase. . . . . . . . . . 20

13 Different stability margins when inertial, elastic and manipulation effects arise as a 20- $\mathrm{N}$ constant force opposing motion on horizontal terrain (CASE

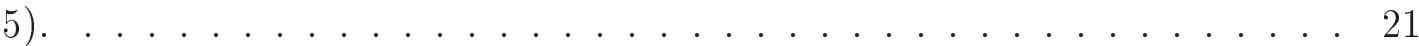

14 Different stability margins when inertial, elastic and manipulation effects arise as a $20-\mathrm{N}$ constant force opposing motion on terrain inclined $10^{\circ}$ from the horizontal plane $($ CASE 6$) . \ldots \ldots \ldots . \ldots \ldots 22$ 
15 Difference between instants of maximum stability for several terrain inclinations $(\alpha)$ and manipulation forces $\left(F_{M}\right)$. (a) SSM and optimum criterion. (b) FASM and optimum criterion. . . . . . . . . . . 24 